1 Universidade Estadual do Oeste do Paraná (Unioeste) - Foz do Iguaçu (PR), Brasil.

tainaabender@gmail.com

2 Universidade Estadual de Londrina (UEL) - Londrina (PR), Brasil.

\section{Rede Mãe Paranaense: análise da estratificação do risco gestacional em três regionais de saúde em $2017-2018$}

\author{
Rede Mãe Paranaense: analysis of the gestational risk stratification in \\ three regional health care in 2017-2018
}

Tainá Aparecida Bender $\mathbf{1}$, Adriana Zilly', Helder Ferreira' ${ }^{\mathbf{1}}$ Rosangela Aparecida Pimenta Ferrari², Andrea Ferreira Ouchi França', Rosane Meire Munhak da Silva'

DOI: $10.1590 / 0103-1104202112907$

\begin{abstract}
RESUMO Este estudo busca analisar e comparar a estratificação de risco gestacional em três regionais de saúde do estado do Paraná, inseridas na Rede Mãe Paranaense, e identificar aspectos que fragilizam sua efetividade. Estudo transversal, analítico, desenvolvido por meio de inquérito com 1.270 puérperas 24 horas após o parto, no alojamento conjunto das maternidades. Para análise dos dados, utilizaram-se os programas SPSS Statistics versão 21 e Bioestat 5.3; para comparação da estratificação de risco, o teste Quiquadrado de Pearson ou Exato de Fisher $(\mathrm{p}<0,05)$. A estratificação de risco ocorreu com maior frequência em serviços de Atenção Primária à Saúde, quando comparados à admissão nas maternidades, nas três regionais de saúde. Houve inconsistências no registro e na informação às usuárias sobre identificação de risco, em especial, nas regionais de Londrina e Cascavel. Gestantes de alto risco foram estratificadas como sendo de risco intermediário, principalmente na regional de Londrina. A estratificação de risco proposta pela Rede Mãe Paranaense se encontra incipiente nas regionais de saúde estudadas, podendo fragilizar a identificação de fatores que contribuem para a prevenção da morbimortalidade materna e infantil.
\end{abstract}

PALAVRAS-CHAVE Cuidado pré-natal. Gravidez de alto risco. Enfermagem obstétrica. Atenção Primária à Saúde.

ABSTRACT This study aims to analyze and compare the stratification of gestational risk in three health regions in the state of Paraná, inserted in the Rede Mãe Paranaense, and to identify aspects that weaken its effectiveness. Cross-sectional, analytical study, carried out with of 1.270 puerperal women 24 hours after delivery, in the maternity accommodation. For data analysis, SPSS Statistics version 21 and Bioestat 5.3 were used; to compare the risk stratification, the Pearson's Chi-square or Fisher's Exact test $(p<0.05)$ was used. Risk stratification occurred more frequently in Primary Health Care services, when compared to admission to maternity wards, in the three health regions. There were inconsistencies in the registration and information to users about risk identification, especially in Londrina and Cascavel regions. High-risk pregnant women were stratified as intermediate risk, mainly in the Londrina regional. The risk stratification proposed by Rede Mãe Paranaense is incipient in health regions studied, which may weaken the identification of factors that contribute to prevention of maternal and child morbidity and mortality.

KEYWORDS Prenatal care. High-risk pregnancy. Obstetric nursing. Primary Health Care. 


\section{Introdução}

A Organização Mundial da Saúde (OMS) define mortalidade materna como as mortes ocorridas durante a gestação ou em até 42 dias após o parto'. É considerada um problema de saúde pública, por ser um indicador de saúde importante que reflete a qualidade na atenção à saúde e a realidade social de uma população. Sua incidência é elevada em países em desenvolvimento, sendo vista como um atentado ao direito humano ${ }^{2}$. As principais causas de mortes maternas na atualidade incluem as síndromes hipertensivas, hemorragias, infecções e os abortos provocados ${ }^{3,4}$.

Estimativas da OMS apontam que cerca de 830 mulheres morrem diariamente por problemas relacionadas à gestação e ao parto no mundo - pontua-se que $90 \%$ das mortes maternas poderiam ser evitadas ${ }^{2,5}$. No Brasil, no ano de 2016, em torno de 1.485 mulheres morreram pelas complicações ocasionadas nos períodos da gestação, parto e puerpério ${ }^{6}$. Em 2019, o índice de mortalidade materna foi de aproximadamente 64,5 óbitos para cada 100 mil nascidos vivos, sendo o maior índice encontrado na região Norte do País 4 .

Na década de 1980, estimava-se que cerca de 500 mil mulheres perdiam suas vidas anualmente devido a causas evitáveis relacionadas à gestação. Esforços para prevenir e detectar precocemente as complicações foram iniciados, e o enfrentamento das complicações obstétricas passou a ter maior importância ${ }^{7}$. No Brasil, na mesma década, o sistema de saúde passou a ser único e universal, denominado Sistema Único de Saúde (SUS), o qual buscou aperfeiçoar os programas de saúde formulados anteriormente ${ }^{8}$.

Em 1984, foi criado o Programa de Atenção Integral à Saúde da Mulher (PAISM), buscando incluir a mulher como sujeito ativo no que tange ao cuidado com sua saúde ${ }^{9}$. Entre os princípios do programa, destacam-se a integralidade, equidade, descentralização, hierarquização e regionalização dos serviços de saúde. Ao longo de 20 anos, o PAISM passou por inúmeros desafios e culminou em avanços, sobretudo para o planejamento familiar, mas também permitiu lacunas, como a atenção ao climatério/menopausa, aos problemas ginecológicos, à infertilidade/reprodução assistida, à saúde da mulher adolescente, a atenção a doenças crônico-degenerativas e infectocontagiosas, à mulher trabalhadora, à saúde mental e à inserção de gênero e raça nas ações em saúde ${ }^{10}$.

Ao ter em vista tais lacunas deixadas pelo PAISM, o Ministério da Saúde (MS) do Brasil, em 2004, lançou a Política Nacional de Atenção Integral à Saúde da Mulher (PNAISM), a qual busca resgatar a ênfase na abordagem de gênero e na integralidade como eixo norteador das práticas de cuidado à saúde da mulher"1 . Suas prioridades são: melhorar as condições de vida e saúde das mulheres por meio da ampliação do acesso aos bens e serviços no âmbito do SUS; contribuir para a redução da morbimortalidade materna, com ênfase nas causas evitáveis; e qualificar e humanizar a atenção à saúde da mulher ${ }^{11,12}$.

Para incrementar essas ações, em 2011, o MS instituiu a Rede Cegonha, via Portaria ${ }^{0} 1.459$, com o fito de garantir o direito ao planejamento reprodutivo e à atenção humanizada durante a gestação, parto e puerpério, oferecendo também à criança a garantia do nascimento seguro e do crescimento saudável ${ }^{13}$. Essa rede se organizou com o intuito de promover acesso, acolhimento e resolutividade, mediante a atenção à saúde no momento do parto, nascimento e puerpério, e de um sistema logístico que garantisse o transporte sanitário e a regulação, assegurando o acesso aos serviços de Atenção Primária à Saúde (APS) e maternidades com profissionais qualificados ${ }^{\mathbf{1 2 , 1 4}}$.

Além da instituição de políticas e programas de saúde, para reduzir as taxas de mortalidade materna, é necessário garantir atenção e cuidado de qualidade para a mulher em todo o ciclo gravídico-puerperal, conhecendo sua realidade e suas características de vida, sejam demográficas, socioeconômicas, obstétricas e reprodutivas. A compreensão dessas características auxilia na identificação de forma rápida do risco gestacional, garantindo o encaminhamento correto das gestantes para os serviços de pré-natal mais adequados. Desse modo, é possível promover a saúde materna e garantir o tratamento adequado para cada risco apresentado ${ }^{15-18}$. 
Estratificar o risco gestacional é fundamental para o seguimento à saúde, considerando sua capacidade de reduzir a morbimortalidade materna e infantil por causas evitáveis. A estratificação deve ser realizada desde o início do pré-natal até o momento do parto pelo médico ou enfermeiro e registrada no prontuário e na carteira de saúde da gestante, visto que o atendimento será realizado de forma mais adequada e, consequentemente, contribuirá favoravelmente para a redução dos índices de mortalidade ${ }^{19}$.

Nessa direção e com base na Rede Cegonha, em 2012, no estado do Paraná, foi instituída a Rede Mãe Paranaense (RMP). Essa rede de atenção busca promover ações de atenção materno-infantil, captar precocemente a gestante, garantir acompanhamento durante todo o pré-natal, com recomendação mínima de sete consultas, realizar exames, estratificar o risco gestacional, com direcionamento à unidade especializada para gestantes e crianças que apresentem riscos, além de garantir o parto mediante a interlocução da rede hospitalar conforme o risco apresentado 20-23. A estratificação de risco permite orientar o encaminhamento das gestantes da porta de entrada para o pré-natal aos serviços de APS e aos serviços especializados ${ }^{16,24}$.

A partir da RMP, o risco gestacional foi organizado em habitual, intermediário e alto. Consideram-se como gestantes de risco habitual aquelas que não apresentam fatores de risco individual, sociodemográficos, histórico de reprodução anterior ou doenças. No risco intermediário, incluem-se as gestantes negras ou indígenas, com idade maior que 40 anos, analfabetas ou com histórico escolar menor que três anos e com histórico de óbito em gestação anterior. Já o alto risco envolve gestantes que apresentam doenças prévias, como hipertensão arterial e/ou diabetes, com intercorrências clínicas e obstétricas durante a gestação atual, como hipertensão arterial, gestação gemelar, trabalho de parto prematuro, entre outras ${ }^{23,24}$.

Segundo estudo realizado com enfermeiros atuantes em serviços de APS no Paraná, a estratificação de risco gestacional deve ser realizada em todas as consultas, e, no início do pré-natal, o enfermeiro é o profissional responsável por essa estratificação $0^{25}$. Outro estudo, também realizado no estado do Paraná, mostrou que na maioria das unidades de APS não há estratificação do risco gestacional no início do pré-natal, conforme preconiza a RMP26. Dessa maneira, a estratificação do risco gestacional nesse estado permanece insipiente, considerando que há falhas em sua realização ainda na primeira consulta de pré-natal.

Por se entender a importância da estratificação do risco gestacional na redução da morbimortalidade materno-infantil e por verificar que são necessários mais estudos para compreender os desafios à sua realização com a implantação da RMP, na presente investigação, o objetivo é analisar e comparar a estratificação de risco gestacional em três regionais de saúde do estado do Paraná, inseridas na RMP, e identificar aspectos que fragilizam sua efetividade.

\section{Material e métodos}

Realizou-se um estudo transversal, analítico, envolvendo maternidades que atendem gestantes de risco habitual, intermediário e alto, pertencentes às Regionais de Saúde (RS) de Foz do Iguaçu ( $9^{\text {a }}$ $\mathrm{RS})$, Cascavel ( $\left.10^{\mathrm{a}} \mathrm{RS}\right)$ e Londrina ( $\left.17^{\mathrm{a}} \mathrm{RS}\right)$.

O cálculo amostral da população de cada maternidade foi empreendido com base no número de partos do ano de 2016, considerando $\mathrm{N}$ - tamanho (número de elementos) da população; $\mathrm{n}$ - tamanho (número de elementos) da amostra; n0 - uma primeira aproximação para o tamanho da amostra; e E0 - erro amostral tolerável. Por se conhecer o tamanho da população, efetuou-se o cálculo amostral na seguinte expressão: $\mathrm{n} 0=1 /(\mathrm{E} 0) 2 \cdot 0,05=400$, o qual foi corrigido por: $\mathrm{n}=\mathrm{N} . \mathrm{n} 0 / \mathrm{N}+\mathrm{n} 0$. Considerou-se uma margem de erro da pesquisa de $5 \%$ e nível de confiança de $95 \%$.

Foram incluídas as puérperas internadas no alojamento conjunto das referidas maternidades, independentemente da estratificação de risco realizada. Excluíram-se as puérperas que apresentaram problemas de saúde mental registrados em prontuário. Dessa forma, 
participaram da pesquisa 1.270 puérperas, sendo 397 da RS de Foz do Iguaçu, 385 da RS de Cascavel e 488 da RS de Londrina.

Os dados foram coletados por acadêmicos do quarto e quinto anos do curso de graduação em Enfermagem e por pós-graduandos de programas da área da saúde de instituições públicas de ensino, os quais foram treinados por enfermeiros com experiência profissional e acadêmica na área da saúde materno-infantil. A coleta aconteceu no segundo semestre de 2017 e primeiro de 2018, mediante inquérito, utilizando-se um instrumento estruturado. Houve a necessidade de coleta de dados documentais nos prontuários e na carteira de saúde da gestante, e, em seguida, aplicou-se o questionário com as puérperas, após 24 horas do nascimento da criança, em local privativo, com duração média de 45 minutos, sem a presença de familiares ou profissionais de saúde das referidas maternidades.

O instrumento foi organizado pelas autoras e analisado por três especialistas, com a finalidade de identificar os aspectos sociodemográficos, antecedentes clínicos, história da gestação anterior, assistência pré-natal na gestação atual e condições da parturiente na admissão na maternidade de cada mulher inserida na pesquisa.

Os dados foram tabulados no Microsoft Excel ${ }^{\circledR}$. Para a análise estatística, foram utilizados os programas SPSS Statistics versão 21 e Bioestat 5.3. A comparação da estratificação de risco realizada nas RS e as categorias de respostas das variáveis estudadas foram realizadas com os testes Quiquadrado de Pearson ou Exato de Fisher. Na análise estatística, adotou-se o nível de significância $\alpha=0,05$.

O projeto de pesquisa foi submetido e aprovado pelo Comitêde Ética em Pesquisa da Universidade Estadual de Londrina, sob o número de Parecer 2.053.304, CAAE: 67574517.1.1001.5231, o qual segue os critérios indicados pela Resolução $n^{\circ}$ 466/12, que envolve pesquisa com seres humanos. Todas as participantes assinaram o Termo de Consentimento Livre e Esclarecido, sendo garantidos o anonimato e o sigilo das informações de cada indivíduo.

\section{Resultados}

Entre as 1.270 participantes do estudo, 728 foram estratificadas como de risco habitual, 166 como risco intermediário e 289 como alto risco nos serviços de APS. Para as demais participantes (87), não foi realizada a estratificação de risco.

A análise de resíduos do Teste Qui-quadrado apontou que a RS de Londrina apresentou maior estratificação do risco intermediário, estatisticamente significativo $(p<0,001)$. Do mesmo modo, na regional de Cascavel, foi mais frequente a estratificação do alto risco, como se observa na tabela 1 .

Tabela 1. Estratificação do risco gestacional por unidades de atenção primária nas Regionais de Saúde de Foz do Iguaçu, Cascavel e Londrina, PR, 2017-2018

\begin{tabular}{lrrrrrr}
\hline Classificação & Foz do lguaçu $(\mathbf{n = 3 9 7 )}$ & \multicolumn{2}{r}{ Cascavel $(\mathbf{n = 3 8 5 )}$} & \multicolumn{2}{r}{ Londrina $(\mathbf{n}=\mathbf{4 8 8})$} \\
& $\mathbf{n}$ & $\mathbf{\%}$ & $\mathbf{n}$ & $\mathbf{\%}$ & $\mathbf{n}$ & $\mathbf{\%}$ \\
\hline Risco Habitual & 242 & 61,0 & 222 & 57,7 & 264 & 54,1 \\
Risco intermediário & 50 & 12,6 & 12 & 3,1 & 104 & 21,3 \\
Alto Risco & 75 & 18,9 & 119 & 30,9 & 95 & 19,5 \\
Não estratificado & 30 & 7,5 & 32 & 8,3 & 25 & 5,1 \\
Valor de $p$ & & & & & & 0,001 \\
\hline
\end{tabular}

Fonte: Elaboração própria. 
A tabela 2 indica que a realização da estratificação do risco gestacional pelos serviços de APS ocorreu em maior número na RS de Londrina e em menor número na RS de Cascavel.

No tocante à informação sobre o risco gestacional, existe uma diferença estatística sobre a comunicação da estratificação de risco durante o pré-natal. Pela análise de resíduos ajustados, a não informação da estratificação na RS de Foz do Iguaçu e Londrina foi significante.

Com relação ao profissional que informou a estratificação, verificou-se uma diferença pelo teste exato de Fisher. $\mathrm{Na}$ análise de resíduos ajustados, os maiores valores foram referentes ao profissional enfermeiro na RS de Foz do Iguaçu; e na RS de Cascavel, ao médico.

Observou-se diferença significativa quanto ao registro do alto risco entre as regionais nas causas dos riscos registrados. A análise de resíduos apontou que os dados que mais influenciaram o resultado significativo do teste foram a realização do registro de risco pela
RS de Foz do Iguaçu e a falta de registros na RS de Cascavel.

No que tange à estratificação de risco durante o pré-natal, foi possível observar, por meio da análise de resíduos ajustados, que houve maior influência no resultado quanto à estratificação de forma adequada na RS de Cascavel e inadequada na RS de Londrina.

Além disso, foi possível analisar, de acordo com o Teste Qui-quadrado, os maiores valores que se encontram para a ocorrência de erros na estratificação relativos ao risco intermediário, o qual deveria ter sido classificado como alto risco nas três RS. Já o teste de Fischer apresenta uma diferença estatística quanto à correta estratificação de risco entre as regionais durante o pré-natal. De acordo com a análise de resíduos ajustados, os valores que mais contribuíram para o resultado foram a estratificação adequada na RS de Cascavel e a estratificação inadequada na RS de Londrina.

Tabela 2. Aspectos da estratificação do risco gestacional em serviços de Atenção Primária à Saúde nas Regionais de Saúde de Foz do Iguaçu, Cascavel e Londrina, PR, 2017-2018

\begin{tabular}{|c|c|c|c|c|c|c|c|}
\hline & \multicolumn{2}{|c|}{ Foz do Iguaçu } & \multicolumn{2}{|c|}{ Cascavel } & \multicolumn{2}{|c|}{ Londrina } & \multirow[t]{2}{*}{ Valor $p$} \\
\hline & $\mathrm{n}$ & $\%$ & $\mathbf{n}$ & $\%$ & $\mathrm{n}$ & $\%$ & \\
\hline \multicolumn{8}{|l|}{ Estratificado o risco } \\
\hline Sim & 367 & 92,4 & 353 & 91,7 & 463 & 94,9 & \multirow{3}{*}{0,143} \\
\hline Não & 30 & 7,6 & 32 & 8,3 & 25 & 5,1 & \\
\hline Total & 397 & 100,0 & 385 & 100,0 & 488 & 100,0 & \\
\hline \multicolumn{8}{|c|}{ Informada sobre o risco } \\
\hline Sim & 225 & 61,3 & 223 & 63,2 & 284 & 61,3 & \multirow{4}{*}{$0,003^{\star}$} \\
\hline Não & 141 & 38,4 & 123 & 34,8 & 159 & 34,4 & \\
\hline Não registrado & 1 & 0,3 & 7 & 2,0 & 20 & 4,3 & \\
\hline Total & 367 & 100,0 & 353 & 100,0 & 463 & 100,0 & \\
\hline \multicolumn{8}{|c|}{ Profissional que informou } \\
\hline Enfermeiro & 135 & 60,0 & 30 & 13,4 & 105 & 37,0 & \multirow{5}{*}{$0,001 \#$} \\
\hline Médico & 79 & 35,1 & 154 & 69,1 & 145 & 51,1 & \\
\hline Médico/enfermeiro & 4 & 1,8 & 2 & 0,9 & 2 & 0,7 & \\
\hline Não registrado & 7 & 3,1 & 37 & 16,6 & 32 & 11,2 & \\
\hline Total & 225 & 100,0 & 223 & 100,0 & 284 & 100,0 & \\
\hline
\end{tabular}


Tabela 2. (cont.)

\begin{tabular}{|c|c|c|c|c|c|c|c|}
\hline & \multicolumn{2}{|c|}{ Foz do Iguaçu } & \multicolumn{2}{|c|}{ Cascavel } & \multicolumn{2}{|c|}{ Londrina } & \multirow[t]{2}{*}{ Valor $p$} \\
\hline & $\mathrm{n}$ & $\%$ & n & $\%$ & $\mathrm{n}$ & $\%$ & \\
\hline \multicolumn{8}{|c|}{ Causa do Risco intermediário } \\
\hline Com registro & 44 & 88,0 & 8 & 66,7 & 75 & 72,1 & \multirow{3}{*}{$0,053 \#$} \\
\hline Sem registro & 6 & 12,0 & 4 & 33,3 & 29 & 27,9 & \\
\hline Total & 50 & 100,0 & 12 & 100,0 & 104 & 100,0 & \\
\hline \multicolumn{8}{|l|}{ Registro do Alto risco } \\
\hline Com registro & 71 & 94,7 & 87 & 73,1 & 77 & 81,0 & \multirow{3}{*}{$0,001^{\star}$} \\
\hline Sem registro & 04 & 5,3 & 32 & 26,9 & 18 & 19,0 & \\
\hline Total & 75 & 100,0 & 119 & 100,0 & 95 & 100,0 & \\
\hline \multicolumn{8}{|c|}{ Estratificação de risco } \\
\hline Adequada & 335 & 91,3 & 344 & 97,4 & 402 & 86,8 & \multirow{3}{*}{$0,001^{\star}$} \\
\hline Inadequada & 32 & 8,7 & 9 & 2,6 & 61 & 13,2 & \\
\hline Total & 367 & 100,0 & 353 & 100,0 & 463 & 100,0 & \\
\hline \multicolumn{8}{|l|}{ Erros encontrados } \\
\hline $\mathrm{RI}$ devendo ser $\mathrm{RH}$ & 6 & 18,7 & - & - & 2 & 3,3 & \multirow{5}{*}{$0,003 \#$} \\
\hline RI devendo ser AR & 19 & 59,4 & 6 & 66,7 & 55 & 90,2 & \\
\hline AR devendo ser $\mathrm{RH}$ & 1 & 3,1 & - & - & 1 & 1,6 & \\
\hline AR devendo ser RI & 6 & 18,8 & 3 & 33,3 & 3 & 4,9 & \\
\hline Total & 32 & 100,0 & 9 & 100,0 & 61 & 100,0 & \\
\hline
\end{tabular}

Fonte: Elaboração própria.

*Teste Qui-quadrado; \# teste de Fisher.

A tabela 3 apresenta dados relativos à estratificação do risco gestacional na admissão em maternidades das RS estudadas. Para 279 gestantes, não houve a estratificação do risco gestacional. Foi identificada maior estratificação do risco intermediário na RS de Londrina e alto risco na RS de Cascavel, e, ainda, uma falta de estratificação maior na RS de Foz do Iguaçu e menor na RS de Cascavel.

Tabela 3. Estratificação do risco gestacional na admissão em maternidade das Regionais de Saúde de Foz do Iguaçu, Cascavel e Londrina, PR, 2017-2018

\begin{tabular}{lrrrrrr}
\hline & \multicolumn{2}{c}{ Foz do lguaçu $(\mathbf{n = 3 9 7 )}$} & \multicolumn{2}{c}{ Cascavel $(\mathbf{n = 3 8 5 )}$} & \multicolumn{2}{c}{ Londrina $(\mathbf{n}=\mathbf{4 8 8})$} \\
\cline { 2 - 7 } & $\mathbf{n}$ & $\mathbf{\%}$ & $\mathbf{n}$ & $\mathbf{\%}$ & $\mathbf{n}$ & \% \\
\hline Risco Habitual & 182 & 45,8 & 197 & 51,2 & 246 & 50,4 \\
Risco intermediário & 32 & 8,1 & 25 & 6,5 & 75 & 15,4 \\
Alto Risco & 51 & 12,8 & 99 & 25,7 & 84 & 17,2 \\
Não estratificado & 132 & 33,3 & 64 & 16,6 & 83 & 17,0 \\
Valor de $p$ & & & & & & 0,001 \\
\hline
\end{tabular}

Fonte: Elaboração própria. 
Na admissão em maternidades, a realização da estratificação de risco gestacional e a falta de informação sobre o risco foram mais frequentes na RS de Londrina. Sobre a falta de realização da estratificação, os números maiores foram apresentados pela RS de Foz do Iguaçu (tabela 4).

Com relação ao profissional que realizou a estratificação do risco de acordo com o teste de Fischer, destacam-se o enfermeiro na RS de Foz do Iguaçu e a falta de registro do profissional na RS de Cascavel. Em todos os casos, o número de gestantes não informadas sobre o risco foi relativamente alto.

Quanto às causas registradas e não registradas para o risco intermediário, a realização do registro de risco pela RS de Foz do Iguaçu e a falta de registros ocorridos na RS de Cascavel foram significativos; e para o alto risco, a realização do registro ocorreu na RS de Foz do Iguaçu e Londrina; e a falta de registros ocorreu novamente na RS de Cascavel.

De acordo com o Teste Qui-quadrado, a realização adequada da estratificação de risco foi mais relevante na RS de Cascavel, e a realização inadequada em Londrina. Acerca dos erros encontrados, o teste de Fischer apontou que o valor que contribuiu foi o erro de estratificação no risco intermediário, devendo ser alto risco nas três RS.

Tabela 4. Aspectos da estratificação do risco gestacional na admissão nas maternidades nas Regionais de Saúde de Foz do Iguaçu, Cascavel e Londrina, PR, 2017-2018

\begin{tabular}{|c|c|c|c|c|c|c|c|}
\hline & \multicolumn{2}{|c|}{ Foz do Iguaçu } & \multicolumn{2}{|c|}{ Cascavel } & \multicolumn{2}{|c|}{ Londrina } & \multirow[t]{2}{*}{ Valor $p$} \\
\hline & $\mathrm{n}$ & $\%$ & $\mathrm{n}$ & $\%$ & $\mathrm{n}$ & $\%$ & \\
\hline \multicolumn{8}{|l|}{ Estratificado o risco } \\
\hline Sim & 265 & 66,7 & 321 & 83,4 & 405 & 83,0 & \multirow{2}{*}{$0,001^{*}$} \\
\hline Não & 132 & 33,3 & 64 & 16,6 & 83 & 17,0 & \\
\hline Total & 397 & 100,0 & 385 & 100,0 & 488 & 100,0 & \\
\hline \multicolumn{8}{|c|}{ Informada sobre o risco } \\
\hline $\operatorname{Sim}$ & 99 & 37,4 & 155 & 48,3 & 193 & 47,7 & \multirow{3}{*}{$0,001^{\star}$} \\
\hline Não & 166 & 62,6 & 147 & 45,8 & 194 & 47,9 & \\
\hline Não registrado & - & - & 19 & 5,9 & 18 & 4,4 & \\
\hline Total & 265 & 100,0 & 321 & 100,0 & 405 & 100,0 & \\
\hline \multicolumn{8}{|c|}{ Profissional que informou } \\
\hline Enfermeiro & 50 & 50,5 & 25 & 16,1 & 78 & 40,4 & \multirow{5}{*}{$0,001 \#$} \\
\hline Médico & 45 & 45,5 & 79 & 51,0 & 87 & 45,1 & \\
\hline Médico/enfermeiro & 1 & 1,0 & 6 & 3,9 & 5 & 2,6 & \\
\hline Não registrado & 3 & 3,0 & 45 & 29,0 & 23 & 11,9 & \\
\hline Total & 99 & 100,0 & 155 & 100,0 & 193 & 100,0 & \\
\hline \multicolumn{8}{|c|}{ Causa do Risco intermediário } \\
\hline Com registro & 27 & 84,4 & 09 & 35,0 & 57 & 76,0 & \multirow[t]{3}{*}{$0,001^{*}$} \\
\hline Sem registro & 5 & 15,6 & 16 & 64,0 & 18 & 24,0 & \\
\hline Total & 32 & 100,0 & 25 & 100,0 & 75 & 100,0 & \\
\hline \multicolumn{8}{|c|}{ Registro do Alto risco } \\
\hline Com registro & 50 & 98,0 & 67 & 67,7 & 76 & 90,5 & \multirow{3}{*}{$0,001^{*}$} \\
\hline Sem registro & 01 & 2,0 & 32 & 32,3 & 08 & 9,5 & \\
\hline Total & 51 & 100,0 & 99 & 100,0 & 84 & 100,0 & \\
\hline
\end{tabular}


Tabela 4. (cont.)

\begin{tabular}{|c|c|c|c|c|c|c|c|}
\hline & \multicolumn{2}{|c|}{ Foz do Iguaçu } & \multicolumn{2}{|c|}{ Cascavel } & \multicolumn{2}{|c|}{ Londrina } & \multirow[t]{2}{*}{ Valor $p$} \\
\hline & $\mathbf{n}$ & $\%$ & $\mathbf{n}$ & $\%$ & $\mathrm{n}$ & $\%$ & \\
\hline \multicolumn{8}{|c|}{ Estratificação de risco } \\
\hline Adequada & 245 & 92,5 & 310 & 96,6 & 361 & 89,1 & \multirow{3}{*}{$0,001^{\star}$} \\
\hline Inadequada & 20 & 7,5 & 11 & 3,4 & 44 & 10,9 & \\
\hline Total & 265 & 100,0 & 321 & 100,0 & 405 & 100,0 & \\
\hline \multicolumn{8}{|l|}{ Erros encontrados } \\
\hline RI devendo ser RH & 6 & 30,0 & - & - & 12 & 27,3 & \multirow{5}{*}{$0,005 \#$} \\
\hline RI devendo ser AR & 10 & 50,0 & 9 & 81,8 & 32 & 72,7 & \\
\hline AR devendo ser RH & 1 & 5,0 & - & - & - & - & \\
\hline AR devendo ser RI & 3 & 15,0 & 2 & 18,2 & - & - & \\
\hline Total & 20 & 100,0 & 11 & 100,0 & 44 & 100,0 & \\
\hline
\end{tabular}

Fonte: Elaboração própria.

${ }^{\star}$ Teste Qui-quadrado; \# teste de Fisher.

\section{Discussão}

Os dados da presente investigação mostram que o número de casos em que o risco não foi estratificado é preocupante, visto que, conforme a RMP, essa ação deveria acontecer com todas as gestantes, seja nas consultas de pré-natal, em serviços de APS, como também na admissão nas maternidades ${ }^{8,23}$.

Nos serviços de APS, a RS de Londrina realizou mais a estratificação. Nessa regional, houve maior frequência de gestantes de risco intermediário, diferentemente da RS de Cascavel, que apresentou menores números de gestantes com risco estratificado e maior ocorrência de alto risco. Nas maternidades, o risco gestacional foi estratificado com maior ocorrência na RS de Cascavel, em especial, para gestantes de alto risco, e menor ocorrência na RS de Foz do Iguaçu.

Cabe ressaltar que a APS deve ser considerada a porta de entrada da rede que orienta o cuidado à gestante para os demais níveis de atenção, independentemente do risco que apresenta, por sua atuação ser mais próxima à comunidade, por conhecer a realidade e as necessidades de seus usuários, garantindo, assim, o recurso assistencial mais adequado e eficiente para conduzir o seguimento gestacional 27,28 .

Identificar o risco de uma gestação permite promover ações que garantam a redução da morbimortalidade tanto materna quanto infantil, consideradas evitáveis ${ }^{\mathbf{2 8 , 2 9}}$. Além disso, a estratificação do risco gestacional é uma estratégia fundamental que garante organizar a assistência de acordo com as necessidades de saúde, oferecendo assistência integral e de qualidade à mulher è̀ criança. Portanto, falhas na estratificação de risco, sejam na falta ou na estratificação inadequada, podem interferir diretamente em maiores taxas de eventos indesejáveis, como o nascimento prematuro e os agravos que podem ser fatais para a gestante e para a criança ${ }^{\mathbf{1 8}}$.

Um estudo realizado com médicos no estado do Paraná identificou que esses profissionais conhecem e atuam na RMP; contudo, na estratificação de risco, alguns profissionais referiram utilizar a estratificação proposta pelo MS, ou seja, em baixo e alto risco. Outros profissionais descreveram realizar a estratificação conforme preconiza a RMP, em risco habitual, intermediário e alto, mas relataram 
discordância e dificuldades na realização e no encaminhamento das gestantes de risco intermediário ${ }^{28}$, podendo ocasionar diferentes interpretações nas avaliações de risco das gestantes. Por outro lado, os enfermeiros mostraram conhecer os indicadores e objetivos da RMP, reconhecendo esse programa como uma estratégia para aumentar a qualidade de vida em saúde pública, logo, utilizando somente a estratificação de risco da RMP25.

Nesta investigação, com relação ao profissional que realizou a estratificação e informou sobre o risco, o enfermeiro e o médico tiveram participação importante, respectivamente, na RS de Foz do Iguaçu e Cascavel. Mesmo os números sendo altos, os valores de falta de registro também foram considerados preocupantes. Isso se deve ao fato de estudos apontarem que cabe aos profissionais de saúde conhecer as características demográficas, socioeconômicas e obstétricas da gestante e realizar a identificação precoce dos fatores de risco na gestação para prestar o atendimento adequado $\mathbf{1 5 , 1 6 , 2 2 , 2 3}$. Além de fornecer o melhor atendimento, é direito da gestante ser informada sobre o risco ao qual está exposta, além de ter acesso a um atendimento com avaliação e classificação de risco, qualidade no pré-natal e boas práticas e segurança durante o parto ${ }^{30}$. Ademais, a OMS defende a importância de garantir boas experiências para as mulheres durante a gestação e o parto por meio de cuidados dignos e de respeito, sem violência e discriminação ${ }^{31}$.

De acordo com a RMP, é essencial manter a carteira de saúde da gestante preenchida com as informações principais sobre a gravidez, além de apresentar os riscos observados. É preciso atualizá-la a cada consulta, visto que ela servirá de ligação entre as consultas e os atendimentos posteriores, inclusive na atenção hospitalar. Além disso, permitirá garantir atendimento das necessidades e intercorrências durante a gestação e o parto ${ }^{23}$. Por isso, é crucial realizar a estratificação de risco gestacional em cada consulta de pré-natal e, no momento da admissão na maternidade, dispor de informações à gestante e registrar na carteira de saúde, para que outros profissionais possam ter conhecimento dos riscos apresentados.

As gestantes devem receber atendimento de uma equipe multiprofissional, composta por médicos obstetras, enfermeiros, farmacêuticos, nutricionistas, assistentes sociais, entre outros. Cada profissional deve realizar um plano de cuidado para a gestante, sendo de responsabilidade do obstetra e do enfermeiro garantir o atendimento durante o pré-natal, solicitando exames e fazendo o acompanhamento da gestante, além de identificar os riscos existentes. O farmacêutico e o nutricionista, por seu turno, podem fornecer orientações sobre os medicamentos e alimentos ingeridos durante a gestação e o puerpério. Ao assistente social, cabe a responsabilidade de oferecer informações quanto aos direitos da gestante, orientando sobre o encaminhamento para a unidade hospitalar ${ }^{23,32}$. Ademais, é dever de todos os profissionais orientar a gestante acerca da importância da realização do pré-natal, da amamentação e da vacinação ${ }^{33}$.

Também devem ser oferecidas à gestante atividades educativas, conduzidas por um profissional de nível superior durante a gestação ${ }^{23}$. Um estudo realizado com gestantes consideradas de risco intermediário e alto risco participantes do grupo de atendimento no Centro Regional de Especialidades no Noroeste do Paraná apresentou os seus relatos acerca do atendimento recebido da equipe multiprofissional. Além dos profissionais já citados, houve atendimento por profissionais da psicologia e fisioterapia. Nessa organização da atenção pré-natal, quando questionadas sobre o atendimento da equipe multiprofissional, $14,9 \%$ das gestantes consideraram o atendimento regular, e 76,6\% bom $^{32}$.

Outro fator importante relaciona-se aos índices de risco intermediário e alto risco registrados em todas as regionais estudadas. Esses números revelam que as gestantes estão expostas a fatores que ocasionam riscos para sua saúde e para a criança. No 
risco intermediário, observou-se que os fatores predominantes foram a multiparidade e a idade superior a 35 anos. Já no alto risco, os fatores de maior ocorrência foram os problemas clínicos, como hipertensão arterial sistêmica e diabetes mellitus. Um estudo conduzido na cidade de Maringá, PR, mostrou alta ocorrência de mulheres com dois ou mais fatores de risco, com destaque para tabagismo, as síndromes hipertensivas, infecção do trato urinário de repetição, amniorrexe prematura e sangramento ${ }^{34}$. Se esses fatores forem identificados precocemente, podem ser prevenidos e evitar problemas futuros. Isso porque alguns comportamentos maternos e hábitos de vida que influenciam a gestação podem ser modificados ao longo das consultas, como, por exemplo, o tabagismo ${ }^{34}$.

As situações agravantes durante a gestação que colocam em risco a saúde da mãe e da criança podem ser diagnosticadas de forma precoce durante as primeiras consultas do pré-natal, levando em conta as características individuais e condições sociodemográficas desfavoráveis, história reprodutiva anterior, doença obstétrica na gravidez atual e intercorrências clínicas da gestante ${ }^{35}$. Tais fatores podem, inclusive, predizer se uma gestação vai evoluir com risco ou não, como as síndromes hipertensivas, as quais têm mostrado relação com a idade materna, história pregressa e hábitos de vida ${ }^{23}$. Em países desenvolvidos, a cada 100 gestantes, de 2 a 8 desencadeiam as síndromes hipertensivas ${ }^{36}$, responsáveis por $60 \%$ das mortes maternas obstétricas diretas ${ }^{37}$.

É relevante apontar que a estratificação de modo inconsistente verificada na presente pesquisa, com maior frequência na RS de Londrina, é fator preocupante tanto nos serviços de APS quanto nas maternidades. $\mathrm{O}$ índice de gestantes de alto risco, estratificadas como de risco intermediário, foi expressivo nas três regionais. Assinala-se que a correta estratificação é essencial para o encaminhamento da gestante às instituições de referência, independentemente da esfera de atenção à saúde de que necessite ${ }^{\mathbf{1 8}, \mathbf{3 8}}$. Para gestações de alto risco, há necessidade de referenciar para um serviço que seja composto por Unidade de Terapia Intensiva Adulto (UTI-A), neonatal e pediátrica, ambulatórios para o pré-natal de risco e que ofereçam um trânsito facilitado entre os serviços de saúde, assegurando atendimento adequado ${ }^{38,39}$.

Para garantir que a estratificação de risco gestacional seja realizada de forma apropriada, é necessário seguir as diretrizes atribuídas pela RMP e, dessa maneira, definir a vinculação da gestante ao pré-natal e ao hospital para, assim, prestar atendimento das necessidades e intercorrências durante a gestação e o parto ${ }^{23}$. E embora a RS de Cascavel tenha promovido capacitação profissional para aperfeiçoar sua atuação na RMP, como mostrou um estudo realizado em $2014^{25}$, nesta investigação, essa RS apresentou resultados desfavoráveis referentes ao registro da estratificação de risco gestacional no cartão de saúde da gestante. Esse fato mostra que, apesar de os profissionais de saúde terem bagagem de conhecimento a respeito da estratificação de risco e sua importância para o seguimento nos diversos níveis de atenção, esse conhecimento precisa ser reestruturado a partir de experiências concretas que servem de base para as ações subsequentes, para atender a novas demandas dos usuários.

Ademais, promover a atenção pré-natal de forma eficiente, com melhorias em todo o processo de estratificação de risco, incluindo a estratificação correta, realizada em todas as consultas, com informações aos usuários e registro do risco atribuído a cada gestante, envolve a valorização dos profissionais de saúde, proporcionada mediante ações de educação continuada para o seu aperfeiçoamento, somada à garantia de recursos humanos e estruturais para consolidar as ações e práticas assistenciais ${ }^{39}$. Para isso, é crucial a responsabilização e o compromisso dos gestores em saúde em fortalecer o processo assistencial, considerando que desempenham um importante papel para a efetivação da competência de promoção da saúde do segmento materno-infantil40. 


\section{Conclusões}

A estratificação do risco gestacional preconizada pela RMP, amplamente referenciada como essencial para a tomada de decisão na atenção pré-natal, ainda se encontra incipiente em muitos cenários paranaenses, como na presente investigação. A atribuição das falhas envolveu a falta de estratificação pelos serviços de APS, mas, principalmente, o momento de admissão para o parto nas maternidades, somados à falta de registro e de informações às usuárias sobre o risco identificado. Essas falhas foram encontradas nas três RS, particularmente, a falta de estratificação pela APS na RS de Cascavel e nas maternidades na RS de Foz do Iguaçu, e, também, falta de registro e informações nas RS de Cascavel e Londrina.

Além disso, a estratificação de risco foi realizada de forma frágil, considerando a estratificação de gestantes de alto risco e risco intermediário, especialmente na RS de Londrina, mostrando-se uma lacuna para a efetividade do acompanhamento pré-natal.

Importante destacar que muitos problemas de saúde ou até mesmo a mortalidade materna podem estar associados aos fatores de risco na gestação. Este estudo buscou demonstrar a importância de se realizar a estratificação de risco durante todo o ciclo gravídico-puerperal, observando a atuação dos profissionais no tocante aos saberes técnico-científicos, mas também por meio de ações humanizadas, considerando que a mulher tem o direito de ser informada sobre os possíveis riscos aos quais elas e seus bebês encontram-se expostos.

\section{Colaboradores}

Bender TA (0000-0002-5305-469X)*, Zilly A (0000-0002-8714-8205)*, Ferreira H (0000-0003-0715-8057)*, Ferrari RAP (00000003-0157-7461)* e Silva RMM (0000-00033355-0132)* contribuíram com as seguintes atividades: na concepção e no planejamento, na análise e interpretação dos dados; na elaboração do rascunho ou revisão crítica do conteúdo; e na aprovação da versão final do manuscrito. França AFO (0000-0002-00246428)* contribuiu com as seguintes atividades: na elaboração do rascunho e revisão crítica do conteúdo; e na aprovação da versão final do manuscrito. 


\section{Referências}

1. Organização Mundial da Saúde. CID-10: Classificação Estatística Internacional de Doenças e Problemas Relacionados à Saúde. 2. ed. São Paulo: CBCD; EDUSP; 1997.

2. Silva SCM, Monteiro EA, Freitas WDMFE, et al. Diagnóstico da situação de morte materna. Rev. Bras. Promoç. Saúde. 2019 [acesso em 2020 maio 4]; (32):9259. Disponível em: https://periodicos.unifor.br/RBPS/ article/view/9259/pdf.

3. Dias JMG, Oliveira APSD, Cipolotti R, et al. Mortalidade materna. Rev. Med. Minas Gerais. 2015 [acesso em 2020 jun 2]; 25(2):173-79. Disponível em: http:// www.rmmg.org/artigo/detalhes/1771.

4. Ongaratto S. Mortalidade materna: Brasil está cada vez mais longe da meta internacional. 2019. [acesso em 2020 jun 6]. Disponível em: https://revistacrescer.globo.com/Voce-precisa-saber/noticia/2019/07/mortalidade-materna-brasil-esta-cada-vez-mais-longe-da-meta-internacional. html\#: :text=N\%C3\%9AMEROS\%20NO\%20 BRASIL,os\%20Objetivos\%20do\%20Desenvolvimento\%20Sustent\%C3\%Alvel.

5. Mora-Escobar EG, Cusihuamán-Puma AU, Insfrán MD. Análisis situacional de muertes maternas em Paraguay: alcances Del 2008/2018. Rev. salud publica Parag. 2020 [acesso em 2020 jul 2]; 10(1):10-22. Disponível em: http://scielo.iics.una.py/pdf/rspp/ v10nl/2307-3349-rspp-10-01-10.pdf.

6. Rodrigues ARM, Cavalcante AES, Viana AB. Mortalidade materna no Brasil entre 2006-2017: análise temporal. ReTEP. 2019 [acesso em 2020 jun 2]; 11(1):3-9. Disponível em: http://www.coren-ce.org.br/wp-content/uploads/2020/01/Mortalidade-materna-no-Brasil-entre-2006-2017-an\%C3\%Allise-temporal-final. pdf.

7. Souza JP. A mortalidade materna e os novos objetivos de desenvolvimento sustentável (2016-2030). Rev. Bras. Ginecol. Obstet. 2015 [acesso em 2020 maio 4]; 37(12):549-51. Disponível em: https://www.scielo.br/ pdf/rbgo/v37n12/0100-7203-rbgo-37-12-00549.pdf.

8. Santos DRD, Viera CS, Guimarães ATB, et al. Avaliação da eficácia do Programa Rede Mãe Paranaense. Saúde debate. 2020 [acesso em 2020 maio 4]; 44(124):7085. Disponível em: https://www.scielo.br/pdf/sdeb/ v44n124/0103-1104-sdeb-44-124-0070.pdf.

9. Gonçalves JR, Costa RD. O direito à saúde, à efetividade do serviço e à qualidade no acesso às políticas públicas de atenção à saúde da mulher. Revista JRG de Estudos Acadêmicos. 2019; 2(4):119-42.

10. Salimena AMDO, Ribeiro MOS. Significado da histerectomia para a mulher e suas implicações na assistência de enfermagem. Enferm. Bras. 2019 [acesso em 2020 jun 2]; 18(3):460-466. Disponível em: https:// portalatlanticaeditora.com.br/index.php/enfermagembrasil/article/view/2357/pdf.

11. Jorge HMF, Hipólito MCV, Masson VA, et al. Assistência pré-natal e políticas públicas de saúde da mulher: revisão integrativa. Rev. Bras. Promoç. Saúde. 2015 [acesso em 2020 maio 4; 18(1):140-148. Disponível em: https://periodicos.unifor.br/RBPS/article/ view/2864/pdf.

12. Frank BRB, Toso BRGO, Viera CS, et al. Avaliação da implementação da rede mãe paranaense em três regionais de saúde do Paraná. Saúde debate. 2016 [acesso em 2020 maio 4]; 40(109):163-74. Disponível em: https://www.scielo.br/pdf/sdeb/v40n109/0103-1104sdeb-40-109-00163.pdf.

13. Tajra FS, Pontes RJS, Carvalho FHC, et al. Rede cegonha: avaliação do potencial de oferta de serviços de saúde materno-infantil. SANARE (Sobral, Online). 2019 [acesso em 2020 maio 4]; 18(2):59-68. Disponível em: https://sanare.emnuvens.com.br/sanare/article/view/1375/700.

14. Assis TR, Chagas VO, Goes RM, et al. Implementação da rede cegonha em uma regional de saúde do estado Goiás: o que os indicadores de saúde mostram sobre atenção materno-infantil? Reciis - Rev. Eletron. 
Comum. Inf. Inov. Saúde. 2019 [acesso em 2020 jul 1]; 13(4):843-53. Disponível em: https://www.arca. fiocruz.br/handle/icict/38751.

15. Novaes ES, Oliveira RRD, Melo ES, et al. Perfil obstétrico de usuárias do sistema único de saúde após implantação da rede mãe paranaense. Cienc. Cuid. Saúde. 2015 [acesso em 2020 maio 4]; 14(4):1436-44. Disponível em: http://periodicos.uem.br/ojs/index. php/CiencCuidSaude/article/view/27343/16487.

16. Migoto MT, Oliveira RPD, Silva AMR, et al. Mortalidade neonatal precoce e fatores de risco: estudo caso-controle no Paraná. Rev. Bras. Enferm. 2018 [acesso em 2020 mar 16]; 71(5):2675-83. Disponível em: https:/www.scielo.br/pdf/reben/v7ln5/pt_0034-7167-reben-71-05-2527.pdf.

17. Garcia EM, Martinelli KG, Gama SGND, et al. Risco gestacional e desigualdades sociais: uma relação possível? Ciênc. Saúde Colet. 2019 [acesso em 2020 maio 4]; 24(12):4633-42. Disponível em: https://www.scielo.br/pdf/csc/v24n12/1413-8123-csc-24-12-4633.pdf.

18. Leite VC, Gasquez ADS, Bertoncim KRI, et al. Estratificação de risco em gestantes no pré-natal. Rev. UNINGÁ. 2019 [acesso em 2020 maio 4]; 56(52):18493. Disponível em: http://revista.uninga.br/index. php/uninga/article/view/2160/1912.

19. Teixeira RA, Ferrari RAP, Caldeira S, et al. Cuidado gravídico-puerperal em rede: o vivido de enfermeiros, médicos e gestores. Rev. Bras. Enferm. 2019 [acesso em 2020 jun 2]; 72(supl1):159-66. Disponível em: https:/www.scielo.br/pdf/reben/v72sl/pt_0034-7167-reben-72-s1-0151.pdf.

20. Baggio MA, Pereira FDC, Guimarães ATB, et al. Programa Rede Mãe Paranaense: análise da atenção pré-natal em uma regional de saúde. Cogitare Enferm. 2016 [acesso em 2020 maio 4]; 21(3):1-10. Disponível em: https://revistas.ufpr.br/cogitare/article/ view/45301/pdf.

21. Demitto MDO, Gravena AAF, Dell'Agnolo CM, et al. Gestação de alto risco e fatores associados ao óbito neonatal. Rev. Esc. Enferm. USP. 2017 [acesso em 2020 jun 2]; 51:e03208. Disponível em: https://www.scielo. br/pdf/reeusp/v51/pt_1980-220X-reeusp-51-e03208. pdf.

22. Netto A, Silva RRMD, Santos MF, et al. Mortalidade infantil: avaliação do programa rede mãe paranaense em regional de saúde do Paraná. Curitiba: Cogitare Enferm. 2017 [acesso em 2020 maio 4]; 22(1):1-8. Disponível em: https://revistas.ufpr.br/cogitare/article/view/47634/pdf.

23. Paraná. Secretaria de Estado da Saúde, Superintendência de Atenção à Saúde. Linha Guia da Rede Mãe Paranaense. Paraná: Sesa; 2018. [acesso em 2020 mar 16]. Disponível em: http://www.saude.pr.gov.br/arquivos/File/LinhaGuiaMaeParanaense_2018.pdf.

24. Longhi SAT, Peterlini OLG. Comissão interna de near miss materno em um hospital da rede mãe paranaense no sudoeste do Paraná: implantação, desafios e perspectivas. R. Saúde Públ. Paraná. 2019 [acesso em 2020 maio 6]; 2(supl1):21-30. Disponível em: http:// revista.escoladesaude.pr.gov.br/index.php/rspp/article/view/225/58.

25. Caldeira S, Luz MSD, Tacla MTGM, et al. Ações de cuidado do enfermeiro no programa rede mãe paranaense. REME - Rev. Min. Enferm. 2017 [acesso em 2020 maio 12]; 21:e-992. Disponível em: https://cdn. publisher.gn1.link/reme.org.br/pdf/e992.pdf.

26. Murara KD, Andrade SMD, Salamanca MAB, et al. Avaliação do pré-natal em Estratégia Saúde da Família (ESF) e em Unidade Básica de Saúde (UBS). Curitiba: Braz. J. of Develop. 2020 [acesso em 2020 mar 16]; 6(3):14147-61. Disponível em: https://www. brazilianjournals.com/index.php/BRJD/article/ view/7925/6868.

27. Sampaio AFS, Rocha MJFD, Leal EAS. Gestação de alto risco: perfil clínico-epidemiológico das gestantes atendidas no serviço de pré-natal da maternidade pública de Rio Branco, Acre. Recife: Rev. Bras. Saúde Mater. Infant. 2018 [acesso em 2020 jun 2]; 18(3):56775. Disponível em: https://www.scielo.br/pdf/rbsmi/ v18n3/pt_1519-3829-rbsmi-18-03-0559.pdf. 
28. Bugs TV, Caldeira S, Machineski GG, et al. Percepção, conhecimento e atuação dos médicos no programa rede mãe paranaense. Santa Maria: Saúde. 2016 [acesso em 2020 jun 2]; 42(2):185-94. Disponível em: https://periodicos.ufsm.br/revistasaude/article/view/19576/pdf.

29. Fernandes JA, Campos GWDS. Perfil das gestantes de alto risco e a cogestão da decisão sobre a via de parto entre médico e gestante. Rio de Janeiro: Saúde debate. 2019 [acesso em 2020 jun 2]; 43(121):40616. Disponível em: https://www.scielosp.org/pdf/ sdeb/2019.v43n121/406-416.

30. Brasil. Ministério da Saúde. Portaria de Consolidação $n^{\circ} 3$, de 28 de setembro de 2017. Consolidação das normas sobre as redes do Sistema Único de Saúde. Diário Oficial da União. 28 Set 2017.

31. Villamil MML, Botero MDPA, Guzmán CIC. Atención humanizada del embarazo: la mirada de gestantes que acuden a uma unidad hospitalaria de salud. Enferm. actual Costa Rica (Online). 2020 [acesso em 2020 jul 2]; (38):180-95. Disponível em:http://docs. bvsalud.org/biblioref/2020/04/1090095/art13n38. pdf.

32. Francisqueti V, Teston EF, Costa MAR. Atendimento ambulatorial sob a perspectiva de gestantes. Recife: Rev. Enferm. UFPE. 2017; 11(5):1875-81.

33. Andrade UV, Santos JB, Duarte C. A percepção da gestante sobre a qualidade do atendimento pré-natal em UBS, Campo Grande, MS. Campo Grande: Rev. Psicol. Saúde. 2020 [acesso em 2020 jul 2]; 11(1):53-61. Disponível em: http://pepsic.bvsalud.org/scielo.php?script=sci_arttext\&pid=S2177-093X2019000100004.

34. Novaes ES, Melo EC, Ferracioli PLRV, et al. Risco gestacional e fatores associados em mulheres atendidas pela rede pública de saúde. Cienc. Cuid. Saúde. 2018 [acesso em 2020 jun 2]; 17(3):e45232. Disponível em: http://periodicos.uem.br/ojs/index.php/CiencCuidSaude/article/view/45232/751375138778.
35. Leal RC, Santos CNC, Lima MJV, et al. Complicações materno-perinatais em gestação de alto risco. Recife: Rev. enferm. UFPE. 2017; 11(sup14):1641-49.

36. Zanatelli C, Doberstein C, Girardi JP, et al. Síndromes hipertensivas na gestação: estratégias para a redução da mortalidade materna. Rev. Sau. Int. 2016 [acesso em 2020 jul 2]; 9(17):73-81. Disponível em: https:// core.ac.uk/download/pdf/229765191.pdf.

37. Pereira GT, Santos AAPD, Silva JMDOE, et al. Perfil epidemiológico da mortalidade materna por hipertensão: análise situacional de um estado nordestino entre 2004-2013. Ver Fund Care. 2017 [acesso em 2020 jul 2]; 9(3):653-58. Disponível em: http://www. seer.unirio.br/index.php/cuidadofundamental/article/view/5526/pdf_1.

38. Huçulak MC, Peterlini OLG. Rede Mãe ParanaenseRelato de Experiência. Londrina: Rev Espaço para Saúde. 2014 [acesso em 2020 fev 16]; 15(1):77-86. Disponível em: http://168.194.69.20/index.php/espacosaude/article/viewFile/541/pdf_22.

39. Medeiros FF, Santos IDDL, Ferrari RAP, et al. Acompanhamento pré-natal da gestação de alto risco no serviço público. Brasília: Rev. Bras. Enferm. 2019 [acesso em 2020 jun 2]; 72(supl3):213-20. Disponível em: https://www.scielo.br/pdf/reben/v72s3/pt0034-7167-reben-72-s3-0204.pdf.

40. Garcia ESGF, Bonelli MCP, Oliveira AN, et al. As ações de enfermagem no cuidado à gestante: um desafio à atenção primária de saúde. J. res.: fundam. care. online. 2018 [acesso em 2020 jul 2]; 10(3):863-70. Disponível em: http://www.seer.unirio.br/index.php/ cuidadofundamental/article/viewFile/6255/pdf_1.

Recebido em 31/07/2020

Aprovado em 05/11/2020

Conflito de interesses: inexistente

Suporte financeiro: não houve 